\title{
Improved phase-shifting method for automatic processing of moiré deflectograms
}

\author{
Hector Canabal, Juan Antonio Quiroga, and Eusebio Bernabeu
}

\begin{abstract}
An improved moiré deflectometry phase-shifting technique is presented. A squared grating is used to multiplex the information of the deflections in two orthogonal directions in one image. This procedure avoids the need to rotate the gratings to obtain complete deflection information. However, the use of these gratings makes impossible the application of standard phase-shifting algorithms. Specifically, the problems associated with the nonsinusoidal profile of the moire fringes and the low-modulation areas produced by the square gratings are solved. A modified moiré deflectometry phase-shifting method is designed to deal with these problems. In addition, a method to obtain the zero order of the prismatic effect is developed. The technique configures a complete and automatic method of mapping ray deflections. From them the refractive power maps can be derived. Experimental results obtained with a progressive-addition lens are shown. (C) 1998 Optical Society of America
\end{abstract}

OCIS codes: $120.4120,120.5050,220.4840$

\section{Introduction}

Moiré deflectometry is a well-known technique for mapping ray deflections on the basis of the moiré and the Talbot effects. ${ }^{1,2}$ A typical moiré deflectometer consists of a collimated light beam followed by two gratings. Normally the tested phase object is placed before the gratings, as shown in Fig. 1. In this method the distortion of the moiré deflectogram is proportional to the angular deflection of the incident rays on the deflectometer gratings, denoted by $G 1$ and $G 2$ in Fig. 1.

Usually, for obtaining quantitative information from the deflectogram intensity-based methods are used, e.g., automatic fringe detection ${ }^{3}$ and local fringe-direction measurement. ${ }^{4}$ These methods depend on the number of observed fringes and are highly sensitive to the noise. Also, it is not possible to distinguish between positive and negative deflections.

On the other hand, phase-measurement methods such as the heterodyne readout $\operatorname{method}^{5}$ and phase shifting ${ }^{6}$ have been applied to moiré deflectometry.

The authors are with the Departamento de Óptica, Facultad de Ciencias Físicas, Universidad Complutense de Madrid, Ciudad Universitaria s/n, 28040 Madrid, Spain.

Received 24 February 1998; revised manuscript received 11 June 1998.

0003-6935/98/266227-07\$15.00/0

(C) 1998 Optical Society of America
In this case the calculated phase map is related to ray deflection. Although for these methods at least three images are required for different grating positions, they do not have the disadvantages described above.

However, some problems remain in the moiré deflectometry phase-shifting method. First, if Ronchi gratings are used, the nonsinusoidal profile of the moire fringes produces the appearance of harmonic components in the computed phase map. Second, the zero order of this phase map is unknown, meaning that the measured deflection is known from a constant. Third, to obtain complete deflection information of the tested object, it is necessary to take two measures with different grating orientations (usually perpendicular to each other), thus implying a precise rotation of either the object or the gratings. Finally, the phase measured in the observation plane is deformed by the refractive power of the tested object because it is necessary to perform back ray tracing to link the measurements to the corresponding points on the object.

We present a solution to these problems that configures a complete and automatic method to map ray deflections. This method is used to map the deflection of ophthalmic lenses in which the ray deflection corresponds to the prismatic power, and by differentiation of this power, a map of the refractive power of the lens is obtained. ${ }^{7}$ A comparison between our experimental results and those obtained with a commercial focimeter shows good agreement. 


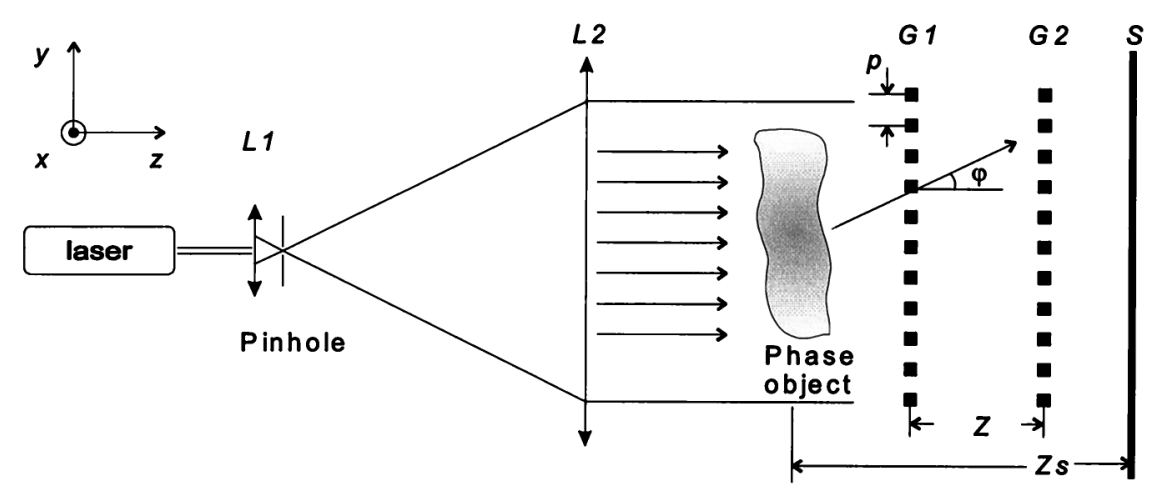

Fig. 1. Experimental setup of the phase-shifting moiré deflectometer. Lenses $L 1$ and $L 2$ expand and collimate, respectively, the laser beam. Gratings $G 1$ and $G 2$ are at a distance $Z$. The screen $S$ is at a distance $Z s$ from the tested object.

\section{Experimental Setup}

The experimental setup is a simple moiré deflectometer similar to that the used in Ref. 6 , and it is shown in Fig. 1. It consists of an expanded collimated He-Ne laser source, followed by two gratings (G1 and G2). The tested lens is placed before the gratings. Different kinds of gratings have been tested: Ronchi, sinusoidal, and squared gratings.

The $G 1$ grating can be moved in its own plane by means of two computer-controlled stepper motors with a step of $0.125 \mu \mathrm{m}$. The $G 2$ grating can be moved along the $z$ axis to adjust the distance $Z$ between the two gratings. For obtaining the maximum fringe contrast this distance is set near a Talbot plane $Z_{k}=k p^{2} / \lambda$, where $p$ is the grating period, $\lambda$ is the wavelength of the monochromatic source used, and $k=1,2,3, \ldots$, is the Talbot plane number.

The moiré fringes are observed through a diffuser screen that produces speckle noise. To minimize the speckle, we rotate the screen. The CCD camera used has square pixels of $7.4 \mu \mathrm{m}$ on a side, so no aspect-ratio considerations are necessary.

\section{Suppression of Harmonic Components}

Commonly in moiré techniques, Ronchi gratings (square transmittance profile) are used. The intensity of the moiré fringes obtained with these gratings in the Fresnel limit is given by ${ }^{8}$

$$
\begin{aligned}
I(x, y)= & \frac{1}{4}+\frac{2}{\pi^{2}} \sum_{n=0}^{\infty} \frac{\cos \left[\pi(2 n+1)^{2} Z^{*}\right]}{(2 n+1)^{2}} \\
& \times \cos \left[2 \pi(2 n+1)\left(\frac{\chi}{p}+\frac{y \theta}{p}\right)\right],
\end{aligned}
$$

where $Z^{*}=Z \lambda / p^{2}$ is the normalized distance between the gratings, $\chi / p$ and $y \theta / p$ are the phase shifts related to the relative translation and rotation, respectively, of the Ronchi rulings, $\chi$ is the offset of the grating line, and $\theta$ is the relative angle between the gratings.

The effect of a phase object introduced in front of the gratings is a distortion in the moire deflectogram. The phase object deflects the ray by an angle $\varphi(x, y)$.
The intensity profile of the deformed moiré fringes (for small $\theta$ and the paraxial approximation) is ${ }^{8}$

$$
\begin{aligned}
I(x, y)= & \frac{1}{4}+\frac{2}{\pi^{2}} \sum_{n=0}^{\infty} \frac{\cos \left[\pi(2 n+1)^{2} Z^{*}\right]}{(2 n+1)^{2}} \\
& \times \cos \left\{(2 n+1) 2 \pi\left[\frac{\chi}{p}+\frac{y \theta}{p}+\frac{Z \varphi(x, y)}{p}\right]\right\} .
\end{aligned}
$$

The phase object introduces a phase shift in the $n$ thorder harmonic component $\phi_{n}(x, y)$ given by

$$
\phi_{n}(x, y)=(2 n+1) \frac{2 \pi Z}{p} \varphi(x, y) .
$$

At separation distances of $Z^{*}=1,2,3, \ldots$, Eqs. (1) and (2) exhibit the well-known triangular wave profile of the moiré fringes. In this situation Pfeifer et $a l .{ }^{6}$ assumed that higher harmonics make little contribution to the entire intensity [as a result of the $1 /(2 n+1)^{2}$ factor] and that the deflection is computed from the fundamental order $(n=0)$ with a simple three-step phase-shifting method. However, in our setup we have noted that the effects of the harmonics components are not negligible. Figure 2(a) shows a reference moiré deflectogram (without lens) in a finite fringe configuration obtained with a linear Ronchi grid of 10 lines $/ \mathrm{mm}$. Figure 2(b) shows a detail of its Fourier transform (obtained digitally with a standard fast Fourier transform algorithm); as can be seen, harmonics up to the fourth order are clearly discernible.

If a standard four-step phase-shifting algorithm is used the effects of the harmonics are not clearly visible but appear in the differentiation of the phase map. For the study of ophthalmic lenses this derivative contains information on the refractive power, so we need to eliminate or at least minimize the effects of the harmonics. A usual solution to this problem is to defocus the CCD objective, but this implies a loss of spatial resolution.

Two solutions for this problem were tested. First, two gratings with a sinusoidal intensity profile were used. The gratings used were commercial sine patterns of 6 lines $/ \mathrm{mm}$. With these gratings the resulting moiré fringe profiles are not longer triangular but 


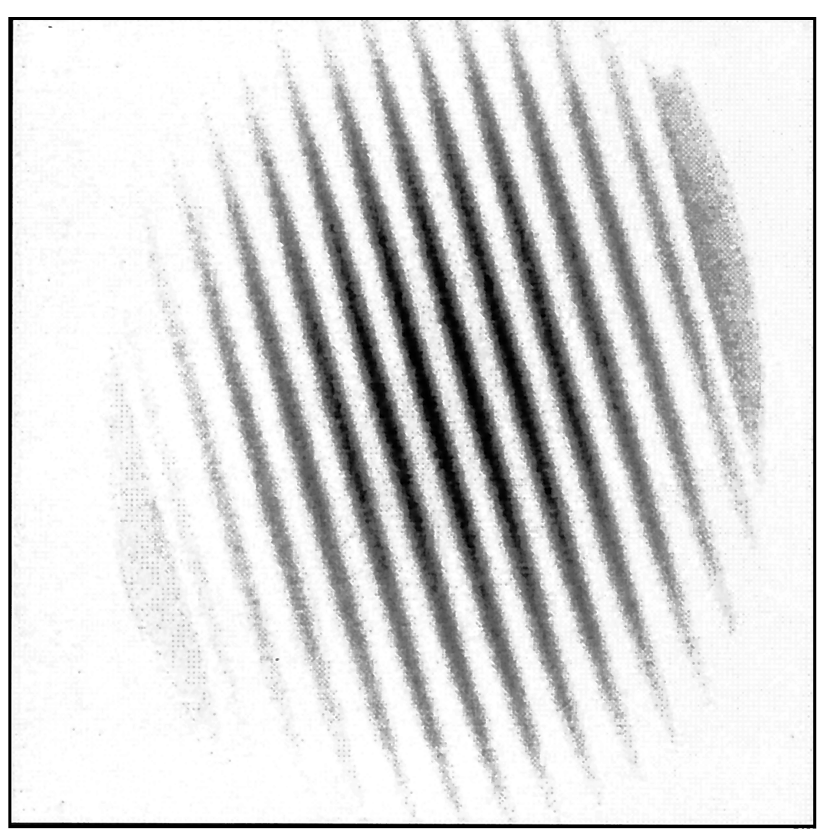

(a)

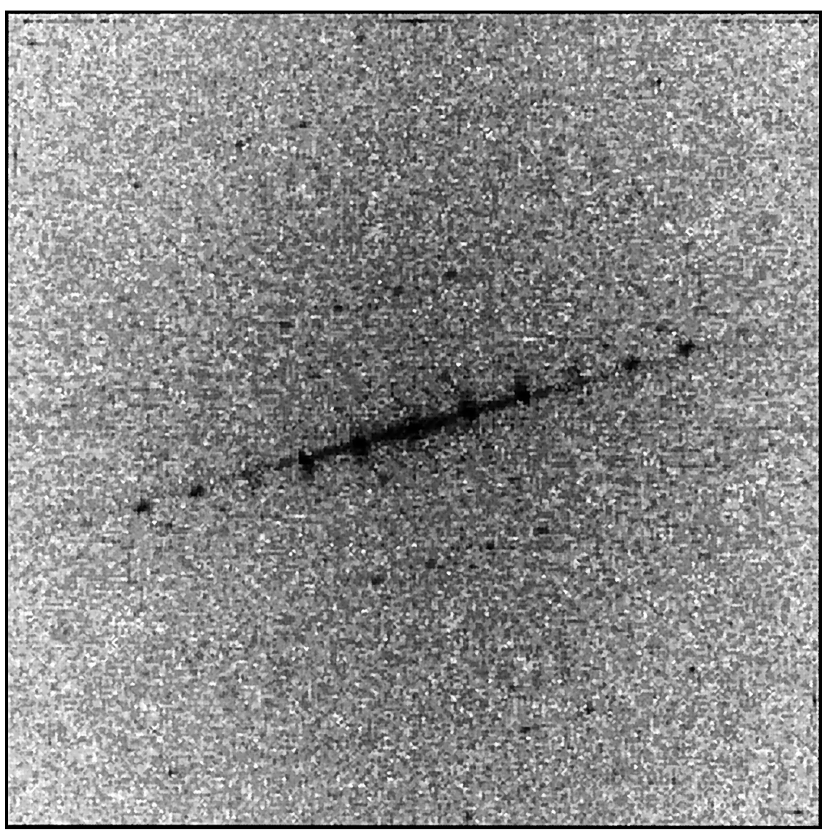

(b)

Fig. 2. (a) Reference moiré deflectogram in a finite-fringe configuration with Ronchi gratings. (b) Fast Fourier transform of (a) with a $2 \times$ zoom. Harmonics up to the fourth order are clearly discernible.

were sinusoidal. In this way the phase map and its derivative do not present distortions resulting from harmonics. Although an ideal solution, the sinusoidal gratings are expensive and difficult to obtain at high frequencies. Moreover, the resultant sinusoidal profile of the fringes has low contrast and can be distorted if the CCD presents nonlinearities.

The second solution is to use phase-shifting algorithms designed for nonsinusoidal waveforms, like

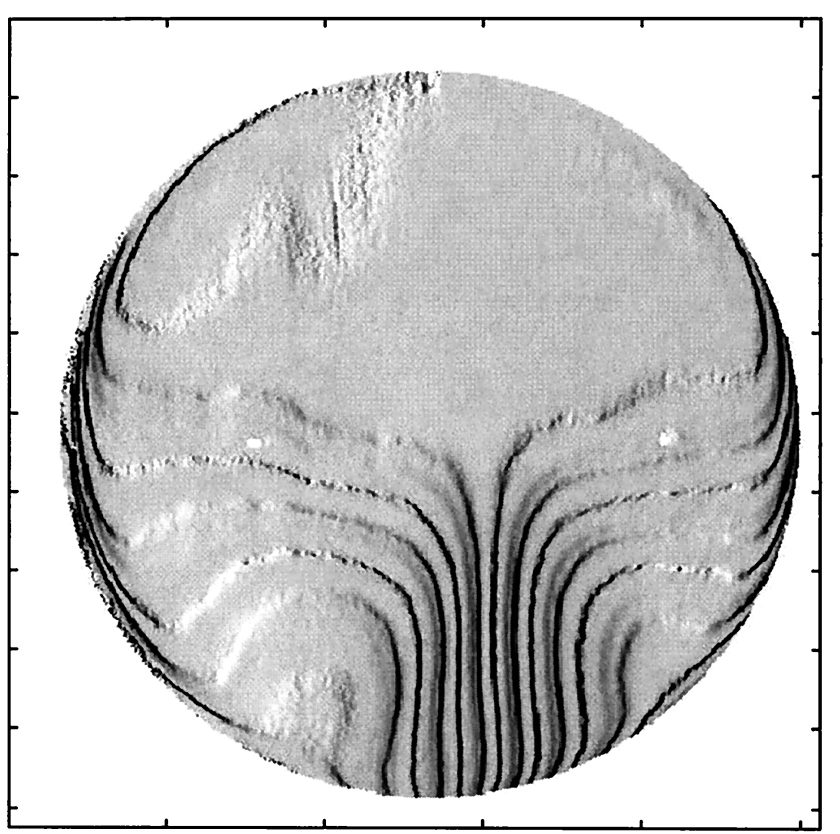

(a)

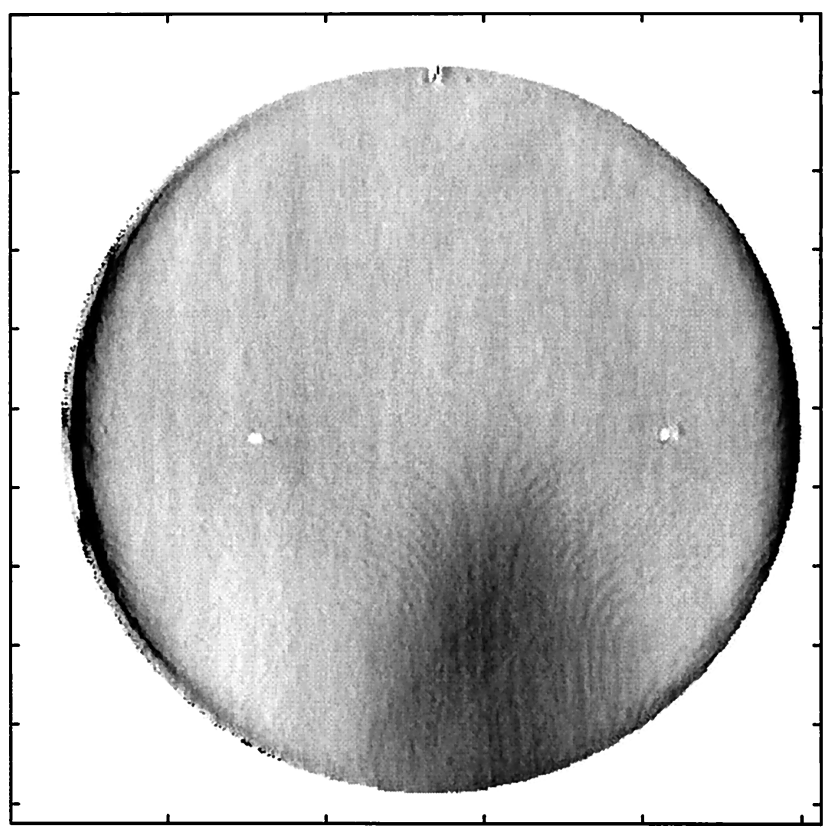

(b)

Fig. 3. (a) Derivative of the phase map of a progressive-addition lens obtained with the four-step algorithm. Information loss is due to the harmonics errors. (b) Derivative of the phase map of the lens of (a) obtained with the 11-step algorithm. The harmonics errors are reduced.

the one presented by Hibino et al. ${ }^{9}$ In that study it was demonstrated that $2 j+3$ samples are necessary for the elimination of the effects of higher-harmonic components up to the $j$ th order in the presence of constant phase-shift error and where the phase-shift interval is $2 \pi /(j+2) \mathrm{rad}$. After different tests the 
11-sample algorithm was selected as a compromise between the sample number and harmonic suppression. This algorithm is designed to suppress up to fourth-order harmonics, which is enough for our experimental configuration. The expression for this phase-shifting algorithm is

$$
\begin{aligned}
& \phi=\arctan \left(\frac{\sum_{i=1}^{11} a_{i} I_{i}}{\sum_{i=1}^{11} b_{i} I_{i}}\right), \\
& a_{i}=\sqrt{3}(0-1-4-7-6067410), \\
& b_{i}=(-2-5-6-18128-1-6-5-2),
\end{aligned}
$$

where $\phi$ is the calculated phase at point $(x, y), a_{i}$ and $b_{i}$ are the sampling amplitudes of the $i$ th sample, and $I_{i}$ is the $i$ th sample's irradiance defined by $I_{i}=I\left(\alpha_{i}\right)$, where $\alpha_{i}=2 \pi i / 6$.

To test the performance of this algorithm, we calculated the deflection map in the $x$-direction lens with a 4-step and the 11-step algorithms. The lens used to illustrate the method is a progressive-addition lens with the addition of $+2.00 \mathrm{D}$ (Selective from the BBGR, Paris). Figures 3(a) and 3(b) show the derivatives of these phase maps. As can be seen from Fig. 3 , the effects of the harmonics have been suppressed in Fig. 3(b), which corresponds to the 11-step algorithm.

\section{Squared Ronchi Gratings and Low-Modulation Areas}

As we said in Section 1, one of the difficulties of moiré deflectometry is the need to rotate accurately either the gratings or the object to obtain the two components of ray deflection. Usually the rotation is done at $90^{\circ}$, and this represents an extra complication in the experimental setup. To avoid this problem, we propose the use of squared Ronchi gratings (SRG's). A SRG can be represented as the superposition of two Ronchi gratings crossed at $90^{\circ}$. To get enough light, we make the first SRG $(G 1)$ a completely black transparency film with small white squares, whereas the second (G2) is the negative of this grating. The SRG presents similar Talbot properties to common Ronchi gratings, although some particularities are to be discussed in a future study.

The moiré pattern obtained with these SRG's has the information of the two components of the ray deflection multiplexed in just one deflectogram. Figure 4 shows the moiré deflectogram of the progressive lens described in Section 3 with the configuration of infinite fringes (the two SRG's are parallel). Note the two sets of fringes corresponding to the $x$ and the $y$ components of the ray deflection. The SRG used was created in POSTSCRIPT and printed directly with a graphics-art photocompositor. The grids have a frequency of 10 lines $/ \mathrm{mm}$ in each principal direction, and the distance $Z$ was set to the first Talbot plane. From Eq. (3) it is shown that each fringe depicted in

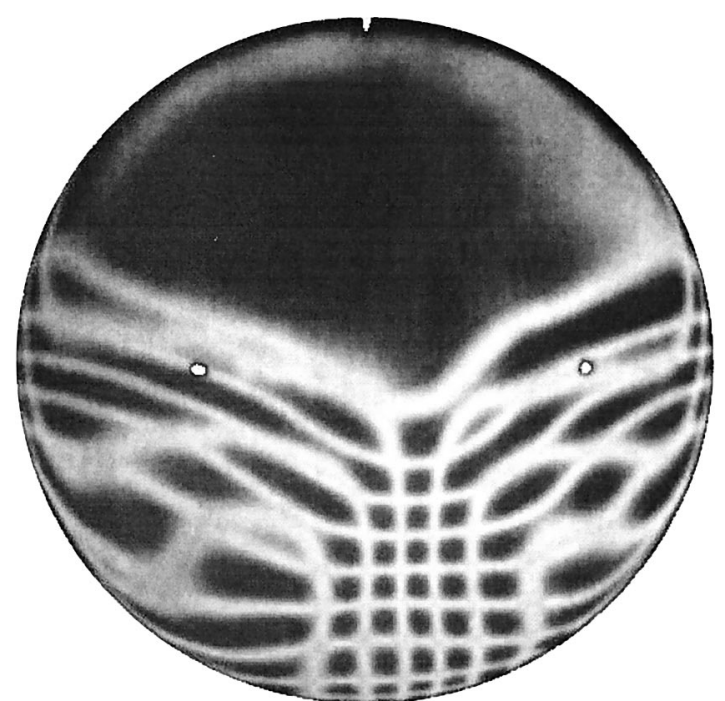

Fig. 4. Moiré deflectogram of the progressive-addition lens in an infinite-fringe configuration with SRG's. Two black points used for position reference can be observed.

Fig. 4 corresponds to a variation of $0.363^{\circ}$ in the ray deflection.

In this configuration, for obtaining the phase map that corresponds to the ray deflection along one of the two principal directions, the $G 1$ grating must be moved normal to the desired direction. So, by moving this grating first in the $x$ and second in the $y$ direction, we obtain the ray deflections in $y$ and $x$, respectively. However, because both fringe patterns are mixed, the phase maps obtained are affected by low-modulation areas that are due to the fringe system associated with the orthogonal deflection component. This situation is similar to the lowmodulation areas that appear in photoelasticity, and we solve it in the same way that Quiroga and González-Cano ${ }^{10}$ did. In fact, the intensity profile of the moiré fringes in infinite-fringe mode obtained with the SRG can be expressed by

$$
\begin{aligned}
I(x, y)= & I_{0}+M \cos \left[2 \pi \frac{Z \varphi_{y}(x, y)}{p}+\alpha\right] \\
& \times \cos \left[2 \pi \frac{Z \varphi_{x}(x, y)}{p}+\beta\right],
\end{aligned}
$$

where $I_{0}$ is the background intensity, $M$ is the overall modulation of the fringe pattern, $\varphi_{x}$ and $\varphi_{y}$ are the deflections in the $x$ and the $y$ directions, respectively, and $\alpha$ and $\beta$ are the phase shifts that are controlled by movement of the $G 1$ grating. To calculate $\varphi_{x}$, we move $G 1$ in the $y$ direction by 11 steps $\alpha_{i}$, as explained in Section 3, whereas the $x$ position of $G 1$ is given by $\beta_{1}$. In this case we get 11 intensity distributions $I_{1 i}$, from which we compute

$$
A_{1}=\sum_{i=1}^{11} a_{i} I_{1 i}, \quad B_{1}=\sum_{i=1}^{11} b_{i} I_{1 i}
$$




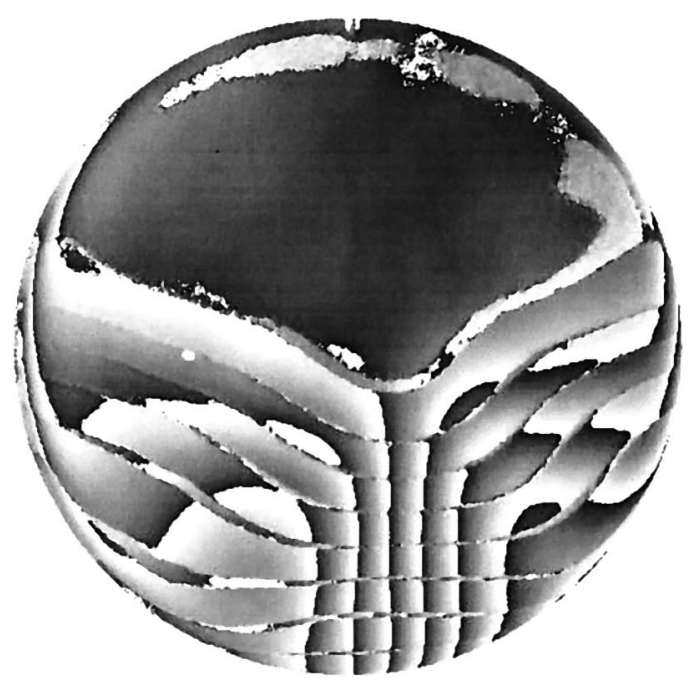

(a)

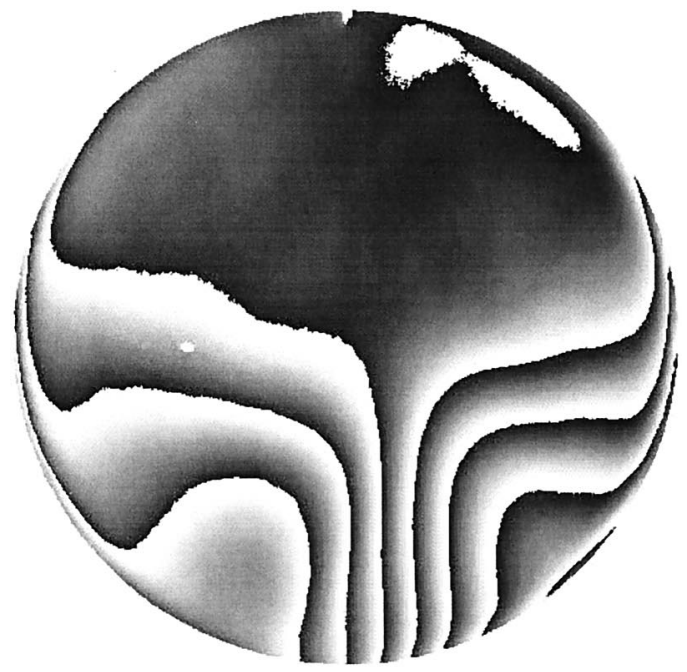

(b)

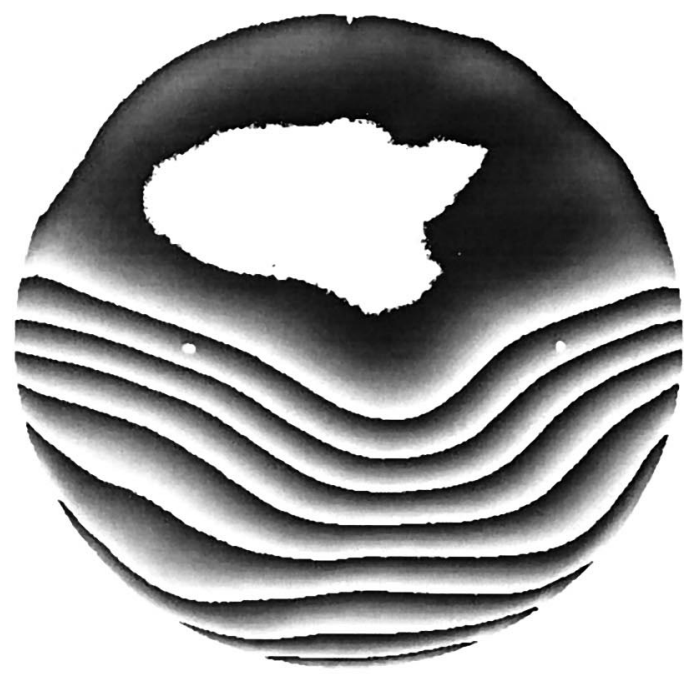

(c)

Fig. 5. (a) Phase map associated with the $x$-deflection component obtained with Eq. (7). The low-modulation areas corrupt the information. (b) Phase map associated with the $x$-deflection component obtained with Eq. (10). The low-modulation areas were with $a_{i}$ and $b_{i}$ defined in Eq. (4). The phase map $\phi_{1 x}$ associated with deflection in the $x$ direction can be calculated by

$$
\phi_{1 x}=\arctan \left(A_{1} / B_{1}\right),
$$

and the modulation of this phase map is

$$
\begin{aligned}
m_{1}(x, y) & =\left(A_{1}{ }^{2}+B_{1}{ }^{2}\right)^{1 / 2} \\
& =\left|M \cos \left[2 \pi \frac{Z \varphi_{y}(x, y)}{p}+\beta_{1}\right]\right| .
\end{aligned}
$$

From Eq. (8), the phase map $\phi_{1 x}$ has lowmodulation areas when the cosine term is near zero, yielding fringe breaks and logical inconsistencies. On the other hand, moving $G 2$ in the $x$ direction in such a way that $\beta_{2}=\beta_{1}+\pi$ generates a second set of intensity distributions, from which $A_{2}$ and $B_{2}$ are computed in the same way as with Eqs. (6). Finally, a second estimation, $\phi_{2 x}=\arctan \left(A_{2} / B_{2}\right)$, is obtained with a modulation given by

$$
m_{2}(x, y)=\left|M \sin \left[2 \pi \frac{Z \varphi_{y}(x, y)}{p}+\beta_{1}\right]\right| \text {. }
$$

In this case the low-modulation areas obtained are associated with regions of low values of the sine term. Because the sine and the cosine are in quadrature, the regions of low values of the cosine term of Eq. (8) coincide with the region of high values for the sine term of Eq. (9). Therefore for every point in the phase map two estimations of the real phase $\phi_{x}$ are obtained. To get the best possible value of the phase estimate, we take a weighted average of the terms $A_{1,2}$ and $B_{1,2}$, with the weights given by the corresponding modulation. ${ }^{10}$ That is, the phase map is computed as

$$
\phi_{x}=\arctan \left(\frac{m_{1} A_{1}+m_{2} A_{2}}{m_{1} B_{1}+m_{2} B_{2}}\right) .
$$

As an example, Fig. 5(a) shows the phase map in the $x$ direction, as obtained with Eq. (7), of the same lens shown in Fig. 4 and also depicts the lowmodulation areas produced by the fringe system associated with deflection in the $y$ direction. Figure 5(b) shows the phase map calculated with Eq. (10). In this figure the improvement with respect to Fig. 5(a) is clearly visible. Finally, Fig. 5(c) shows the phase map $\phi_{y}$ with the low-modulation areas suppressed.

Although prismatic effects are important in the study of an ophthalmic lens, the mapping of the refractive power is more useful for its characterization. Figures 6(a), 6(b), and 6(c) show the derivatives of the phase maps $\phi_{x x}, \phi_{x y}$, and $\phi_{y y}$, respectively. From

suppressed. Each $2 \pi$ jump in the phase map corresponds to a variation of $0.363^{\circ}$ in the ray deflection. (c) Phase map associated with the $y$-deflection component with low-modulation areas suppressed. 


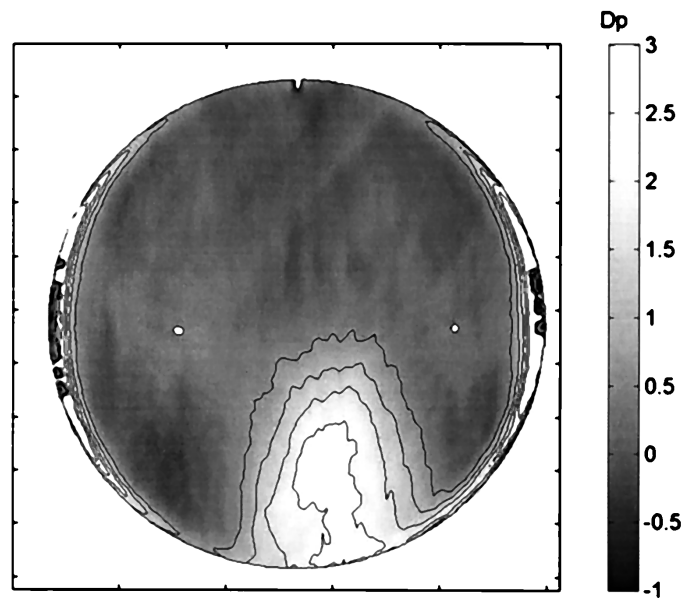

(a)

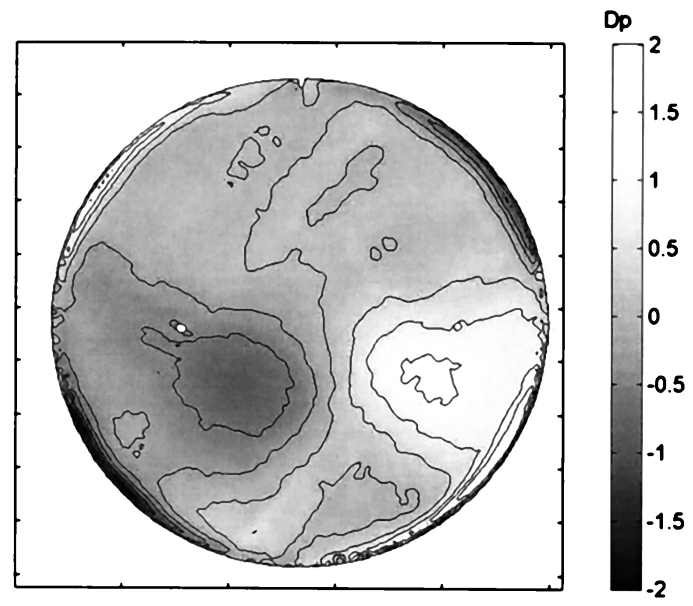

(b)

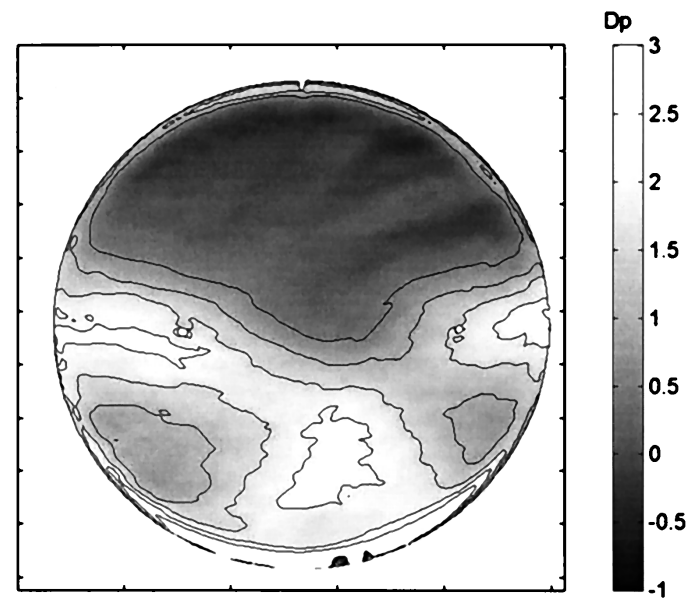

(c)

Fig. 6. Phase derivatives representing (a) the power along the $x$ direction $\phi_{x x}$, (b) torsion $\phi_{x y}$, and (c) the power along the $y$ direction $\phi_{y y}$. The scale is expressed in diopters $(\mathrm{Dp})$.

these phase maps it is simple to calculate the sphere, the cylinder, and the axis, commonly used as figures of merit for the characterization of ophthalmic lenses. ${ }^{7}$
The measurement process requires 22 images to map the deflection in each direction, yielding a total of 44 images for obtaining complete deflection information. Another set of 44 images is needed to subtract the reference information without the lens. The algorithm for acquisition and processing was programmed under the MATLAB environment with a Pentium 166 computer. The complete processing time without any optimization is approximately $7 \mathrm{~min}$.

\section{Calibration of Zero}

As is well known, the phase-shifting techniques are not absolute, that is, a point of known phase must be obtained to realize an absolute measure of the phase. To get this, one can use a commercial focimeter to obtain the prismatic power in a specific point of the lens (for example the center). The drawback of needing a point of known phase can be overcome if the tested object has a point with zero deflection in the field of view. In such a case the zero-deflection points can be detected by use of the standard setup used here. For this two phase maps with slightly different separations $Z$ between the gratings are obtained. That is,

$$
\phi_{\mathrm{I}, \mathrm{II}}(x, y)=\left[\frac{2 \pi}{p} Z_{\mathrm{I}, \mathrm{II}} \varphi(x, y)\right] \bmod 2 \pi .
$$

If the displacement fulfills the condition

$$
\left|Z_{\mathrm{I}}-Z_{\mathrm{II}}\right|<\left|\frac{p}{2} \varphi_{\max }\right|
$$

where $\varphi_{\max }$ is the maximum ray deflection present in the object, these phase maps are equal only at the points with $\varphi(x, y)=0$. Then, from their difference, the zero-deflection points can be located, which makes possible an absolute ray-deflection measurement. Figures 7(a) and 7(b) show the absolute $x$ and $y$-deflection maps obtained by the unwrapping of the phase maps shown in Figs. 5(b) and 5(c), respectively. The solid lines correspond to the areas of zero deflection.

Finally, a comparison of these results with the deflection measured with an automatic focimeter (Humphrey, Model 360) was realized. It must be noted that the deflection information measured with the moiré method is obtained in the observation plane $S$. Therefore a simple ray-tracing technique is applied to obtain the corresponding information in the object plane ${ }^{11}$ and to make possible a comparison with the results obtained with the focimeter. To perform ray tracing, it is necessary to know the distance between the observation plane and the tested object, $Z s$. Figure 8 shows the profile along the principal meridian of the $y$-deflection map shown in Fig. 7(c) as well as the deflection measured with the focimeter along the same meridian. Good agreement between both methods can be seen. 


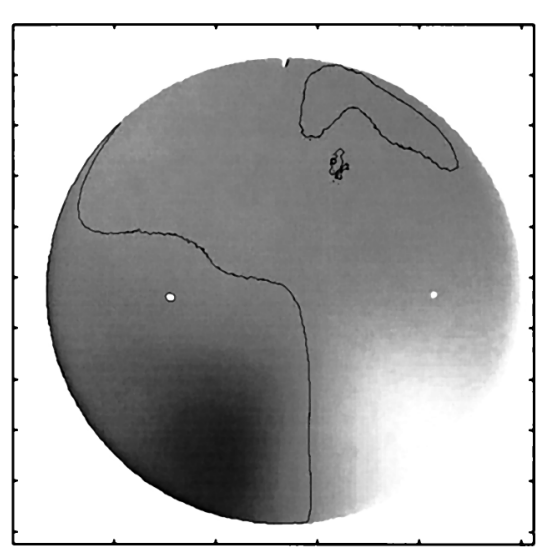

(a)

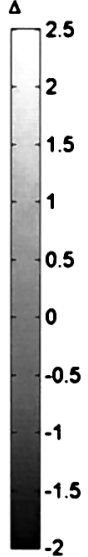

Fig. 7. Absolute ray deflections: (a) $x$ deflection and (b) $y$ deflection in prismatic diopters ( $\Delta$ ). A measure of $1 \Delta$ corresponds to a deflection of $1 \mathrm{~cm}$ in $1 \mathrm{mts}$. The solid lines delineate the zones of zero $x$ and $y$ deflections, respectively.

\section{Conclusions}

We have presented an improved technique for measuring ray deflections by moiré deflectometry phase shifting. The use of squared gratings avoids the need to rotate the gratings to obtain complete deflection information. A modified moiré deflectometry phase-shifting method has been designed to deal with the problems associated with the utilization of these gratings. Finally, a method for obtaining the zero order of the prismatic effect has been developed. The technique constitutes a complete and automatic method for mapping ray deflections and allows information about the local refractive power of the tested object to be obtained.

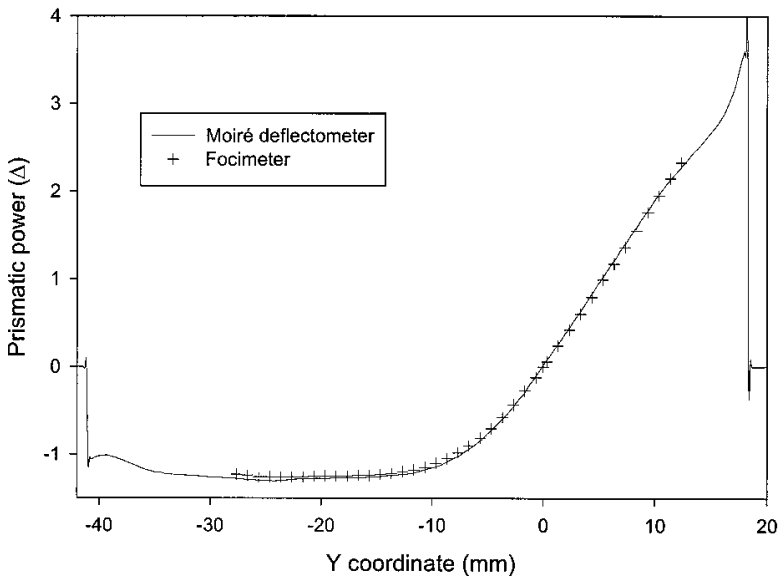

Fig. 8. Profile along the principal meridian (centered vertical line) of the $y$ deflection represented in Fig. 7(b). The solid curve corresponds to the values obtained by moiré deflectometry. The cross points represent the values measured with a commercial focimeter.
We thank J. Alonso, and J. A. Gomez-Pedrero for their useful comments and continuous support during the development of this paper.

\section{References}

1. O. Kafri and I. Glat, "Moiré deflectometry: a ray deflection approach to optical testing," Opt. Eng. 24, 944-960 (1985).

2. O. Kafri and I. Glatt, The Physics of Moiré Metrology (Wiley, New York, 1989).

3. M. Servin, R. Rodriguez-Vera, M. Carpio, and A. Morales, "Automatic fringe detection algorithm used for moiré deflectometry," Appl. Opt. 29, 3266-3270 (1990).

4. H. Canabal, J. A. Quiroga, and E. Bernabeu, "Local fringe direction calculation and application in moiré deflectometry," in Fringe '97, Proceedings of the Third International Workshop on Automatic Processing of Fringe Patterns, W. Jüptner and W. Osten, eds. (Akademie Verlag, Berlin, 1997).

5. J. Striker, "Performance of moiré deflectometry with deferred electronic heterodyne readout," J. Opt. Soc. Am. A 4, 42-50 (1987).

6. T. Pfeifer, B. Wang, J. Evertz, and R. Tutsch, "Phase-shifting moiré deflectometry," Optik (Stuttgart) 98, 158-162 (1995).

7. J. Alonso, J. A. Gómez-Pedrero, and E. Bernabeu, "Local dioptric power matrix in a progressive addition lens," Opthal. Physiol Opt. 17, 522-529 (1997).

8. J. Stricker, "Diffraction effects and special advantages in electronic heterodyne moiré deflectometry," Appl. Opt. 25, 895902 (1986).

9. K. Hibino, B. F. Oreb, D. I. Farrant, and K. G. Larkin, "Phase shifting for nonsinusoidal waveforms with phase-shift errors," J. Opt. Soc. Am. A 12, 761-768 (1995).

10. J. A. Quiroga and A. González-Cano, "Phase-measuring algorithm for extraction of isochromatics of photoelastic fringe patterns," Appl. Opt. 36, 8397-8402 (1997).

11. Y. Hotta, A. Asano, and S. Yokozeki, "Automated Talbot interferometer using moiré fringe scanning and ray tracing," in Proceedings of the Seventeenth General Congress of the International Commission for Optics, Proc. SPIE 2778, 1114-1115 (1996). 